\title{
BEM-DERIVED IMPEDANCE FUNCTIONS FOR 2D INHOMOGENEOUS SOIL MEDIA: AN ENGINEERING PRACTICE CASE STUDY
}

\author{
Georgios A. Chatzistefanou, George D. Manolis* \\ Laboratory for Experimental Mechanics, Department of Civil Engineering, \\ Aristotle University, GR-54124 Thessaloniki, Greece \\ [Received: 16 June 2020. Accepted: 05 October 2020] \\ doi: 10.7546/JTAM.50.20.04.06
}

\begin{abstract}
A methodology is presented here for the determination of complexvalued dynamic impedance functions for arbitrarily shaped embedded strip foundations, by using the displacement-based boundary element method (BEM). More specifically, the formulation incorporates a frequency-dependent fundamental solution obtained in closed form for continuous soil inhomogeneity under plane strain conditions and for viscoelastic material behavior. The recovery of the impedance functions in engineering practice is illustrated via a case study of an instrumented and well-documented public building, whereby the BEM-derived stiffness and damping coefficients are compared to widely used impedance function formulations found in the literature. Finally, the challenges and shortcomings associated with the derivation and use of impedance functions for studying soil-structure interaction (SSI) problems are discussed, where it is pointed out that an effective approach seems to be a re-casting of the BEM formulation as a macro-element to be used in conjunction with a finite element method (FEM) representation of the structure in question.
\end{abstract}

KEY WORDS: impedance functions, boundary element method, embedded foundations, soil-structure interaction, earthquake engineering, inhomogeneous soil profiles.

\section{INTRODUCTION}

The effect of soil-structure interaction (SSI) on the dynamic response of civil engineering structures has been a subject of research for well over five decades. Among the various techniques that have been developed, the substructure method exhibits particular practical interest, since it is the most commonly used approach in engineering analysis and design. Following this approach, the problem is decomposed into two parts, namely the kinematic and inertial interaction parts, in order to determine the foundation input motion and the response of the structure, respectively [1]. The analysis entailed in inertial interaction involves the determination of the foundation's impedance functions, which represent the stiffness and damping coefficients

\footnotetext{
${ }^{*}$ Corresponding author e-mail: gdm @ civil.auth.gr
} 
of the soil continuum, defined at the soil-foundation interface. In general, the derivation of impedance functions for the soil continuum forms a subclass of elastic wave propagation phenomena in geological media [2]. These problems involve a number of important engineering details such as layering, material inhomogeneity, the presence of a liquid phase in the soil, the presence of discontinuities, free-surface relief, etc. Numerical simulations and measurements regarding elastic wave propagation phenomena have clearly demonstrated the importance of all the above factors on the resulting scattered elastic wave fields, and identified the generation of localized stress concentration phenomena [3].

The literature on dynamic SSI is vast and can be traced back to the seminal work of E. Reissner in the 1930's. However, it can be argued that its origins lie on the recovery of fundamental solutions in the $19^{\text {th }}$ century, as pointed out by Kausel in a review of the early history of SSI [4]. Another early state-of-the-art review by Johnson [5] gives a clear picture of the engineering essence of this problem: soil flexibility results in natural period lengthening and damping modification of the soil-structure system. This fact has been the subject of numerous studies utilizing various methodologies [6]. Furthermore, SSI effects can be studied even if the soil impedances are in the form of simple elastic springs. The fact that the problem is time-dependent, however, requires the use of complex-valued impedance functions, where the real part corresponds to a spring, while the imaginary part to a dashpot. In addition, each part must be frequency dependent, since the soil continuum responds differently at low versus high frequencies of vibration. Moreover, there is always a soil mass around the structure that vibrates in tandem with the foundation, giving rise to the concept of added (or virtual) mass. Finally, given the complex composition of the ground formations, the impedance functions should,in theory, be able to account for soil inhomogeneity, anisotropy, the presence of geological cracks and other surface or subsurface discontinuities, plus the possibility for soil nonlinearities and for liquefaction if the level of ground shaking is high. Thus, the problem is practically intractable due to the high complexity manifested by the soil continuum, but this has not prevented the development and use of impedance functions as a means of producing acceptable engineering solutions.

As previously mentioned, the methods used for the recovery of impedance functions started with analytical solutions in the 1930's by E. Reissner (see [7]) and can be classified in three broad categories, namely (i) the use of closed form solutions in the form of published charts and tables [8-10], (ii) simplified strength of materials approaches [11,12] and (iii) various numerical methods, with a pre-eminent method being the various boundary element method (BEM) formulations. For instance, frequency domain BEM formulations for solving SSI problems exhibiting viscoelasticity have been introduced by Manolis and Beskos in connection with the differential 
operator form of the constitutive equations [13], by Kobayashi and Nishimura in connection with the integral form of the constitutive equations [14], and by Abascal and Dominguez in connection with the simple hysteretic damping model [15]. Among much work done on the dynamic response of foundations using the BEM, we only mention the papers by Kokkinos and Spyrakos on strip foundations [16], by Karabalis and Rizos on 3D foundations [17] and by Qian and Beskos on foundation interaction effects [18]. Recently, numerous studies have been published that utilize various BEM formulations for the recovery of impedance functions, modeling complex soil configurations, as for instance by considering layered, transversely isotropic media representations [19]. Also, the non-linear soil behavior can be taken into account by using equivalent linear approaches, since the soil material parameters are dependent on the level of strains which develop in the soil continuum [20]. The reason for the popularity of the BEM in solving problems with semi-infinite boundaries is twofold: (i) These techniques require surface discretization only, thus reducing the dimensionality of the problem by one; (ii) the use of analytical singular solutions of elastodynamics as kernel functions in the BEM formulation renders the method highly accurate by satisfying the radiation (or Sommerfeld) boundary condition.

In this work, we use the BEM to recover soil impedance functions that encompass the time harmonic response of a foundation to prescribed unit displacements, in the form of stiffness and damping functions, for soil media exhibiting inhomogeneity. The soil is modeled as a single-phase material with viscoelastic mechanical properties and a continuous variation of the shear modulus with depth. The foundation is embedded in the soil and can be treated as either rigid or flexible, depending on the definition of the boundary-value problem (BVP) at hand. Furthermore, in order to illustrate the use of impedance functions in engineering practice, a case study of a well-documented municipal building in the island of Lefkas, Greece is examined and comparisons between the proposed methodology and widely used impedance function solutions available in the literature $[8,21]$ are performed.

\section{Mathematical Formulation of the Problem}

In general, a mechanical model appropriate for SSI problems comprises a finite local geological inclusion with discontinuities, embedded in a stack of soil layers, which in turn rest on bedrock [5]. To this end, we present a BEM formulation for BVP that leads to the recovery of impedance functions with the possibility of incorporating as many of the above features as possible. This formulation is for $2 \mathrm{D}$, plane strain cases with frequency-dependent material parameters that allow for viscoelastic behavior based on the correspondence principle of viscoelasticity [13]. Furthermore, by using Bardet's model [22], which represents saturated poroelastic soils as singlephase, Kelvin-Voigt viscoelastic materials, it is possible to model poroelastic media 
at low frequencies of vibration. In addition, the half-plane may have an inhomogeneous structure, whereby the stiffness modulus and the density may continuously vary with depth from the free surface downwards. Of course, there are additional aspects regarding the soil that can be considered, such as anisotropy and the presence of geological cracks, but these render the problem quite complicated, require case-by case examination, and it may not be possible to assign numerical values to all the necessary problem parameters [3].

By using the Cartesian coordinate system $\mathrm{O}-X Y Z$ of Fig. 1, we model a geological region with an external boundary $S$ that is swept by time harmonic elastic waves with a frequency $\omega$. At first, the material is homogeneous and isotropic, while its mechanical properties are described by the Lam constants $\lambda$, $\mu$, by Poisson's ratio $\nu$ and by the mass density $\rho$. In order to account for material viscosity, the Lamé constants are now complex variables in the form $\lambda_{V}=\lambda(1+i \omega \eta), \mu_{V}=\mu(1+i \omega \eta)$, where $\eta$ is the coefficient of viscosity with values in the neighborhood of $1 \%$. Next, the soil material is now considered as continuously inhomogeneous in terms of the spatial variable $\boldsymbol{x}=(x y z)$ in the form

$$
\mu(\boldsymbol{x})=\mu_{0} h(\boldsymbol{x}), \quad h(x)=(a \boldsymbol{x}+b)^{2},
$$

where $a, b$ are constants and the Poisson's ratio value is fixed as $\nu=0.25$. The same variation is assumed for the remaining two material parameters $\lambda(\boldsymbol{x}), \rho(\boldsymbol{x})$. Obviously, if the above constants assume values of $a=0, b=1$, the elastic homogenous case is recovered. The governing equations of motion in the frequency domain, written in terms of the displacement vector $u_{i}$ and the stress tensor $\sigma_{i j}$, and in the absence of body forces, are

$$
\sigma_{i j, j}(\boldsymbol{x}, \omega)+\rho(\boldsymbol{x}) \omega^{2} u_{i}(\boldsymbol{x}, \omega)=0 .
$$

The second term in the above equation represents inertia forces, while the stress equilibrium operator is

$$
\sigma_{i j, j}(\boldsymbol{x}, \omega)=\left\{\lambda(\boldsymbol{x}) u_{k, k}(\boldsymbol{x}, \omega)\right\}_{, i}+\left\{\mu(\boldsymbol{x})\left(u_{i, j}(\boldsymbol{x}, \omega)+u_{j, i}(\boldsymbol{x}, \omega)\right)\right\}_{, j} .
$$

For plane strain conditions, the spatial variable is $\boldsymbol{x}=(x, z)$ and indices $i j k$ all range from 1,2 . Also, the summation convention is implied in the above equations for repeated indices. The reference values for the material parameters are defined at the free surface as $\mu_{0}, \lambda_{0}, \rho_{0}$ and are functions of depth, attaining the values of $\mu_{1}, \lambda_{1}, \rho_{1}$ at $z=d$. The equations of motion (2) form an elliptic system of partial differential equations (PDE), except along line $z=-b / a$, where they degenerate. Next, it is possible to introduce an algebraic transformation for the displacement vector of the type

$$
u_{i}(\boldsymbol{x}, \omega)=h^{-0.5}(\boldsymbol{x}) U_{i}(\boldsymbol{x} \omega),
$$


where $U_{i}$ is now a displacement solution for the dynamic equilibrium equations expressed in terms of the equivalent homogenous medium case as

$$
\mu U_{i, j j}+2 \mu U_{j, i j}+\rho \omega^{2} U_{i}=0 .
$$

A fundamental solution $U_{i j}^{*}(x \xi)$ can now be derived for Eq. (5) for a right-hand side in the form of a point impulse $-h^{-0.5}(\boldsymbol{\xi}) \delta(\boldsymbol{x}-\boldsymbol{\xi})$, where $\delta$ is the Dirac delta function and with $\boldsymbol{x}, \boldsymbol{\xi}$ being receiver and source points, respectively [23]. Carrying on with the algebraic transformation of Eq. (4), the fundamental solution to the original PDE of Eq. (2) is now $u_{i j}^{*}(x, \boldsymbol{\xi})=h^{-0.5}(\boldsymbol{x}) \bullet U_{i j}^{*}(\boldsymbol{x} \boldsymbol{\xi}) \cdot h^{+0.5}(\boldsymbol{\xi})$.

Once the displacement fundamental solution has been recovered, the BEM formulation becomes routine [13]: Starting with the constitutive law, the corresponding traction fundamental solution $T_{i j}^{*}$ can be derived. Then, application of a reciprocity relation between two elastic states in the continuum for the displacements $u_{i}$ and the tractions $t_{i}$, where one state is the scattered wave field (superscript $s c$ ) and the other comprises fundamental solutions for the full space (superscript $*$ ), gives a boundary integral equation in the form

$$
c_{i j} u_{j}^{s c}(\boldsymbol{\xi}, \omega)=\int_{S} U_{i j}^{*}(\boldsymbol{x}, \boldsymbol{\xi}, \omega) t_{j}^{s c}(\boldsymbol{x}, \omega) d S-\int_{S} T_{i j}^{*}(\boldsymbol{x}, \boldsymbol{\xi}, \omega) u_{j}^{s c}(\boldsymbol{x}, \omega) d S .
$$

In the above, both the receiver and source points are defined at the surfaces $S$ of the elastic body in question. We note here that the complete elastodynamic statement given by Eq. (6) involves the total wave field, which is the sum of incident [24] plus scattered wave fields. In our case, there is no incident field as the BVP involves imposed displacements at the soil-foundation interface. Thus, tractions are defined as $t_{i}^{s c}=\sigma_{i j}^{s c} n_{j}$, where $n_{j}$ is the outward pointing, unit normal vector at the surface. Also, $c_{i j}$ is a jump term equal to $0.5 \delta_{i j}$ for smooth surfaces, while the above integrals are understood in a Cauchy-principal value sense. Standard nodal collocation schemes using three-node, curvilinear boundary elements with quadratic polynomial shape functions, renders the BEM integral equations into a system of algebraic equations of dimension $N X N$, where $N$ is the total number of degrees of freedom (DOF) of the problem at hand

$$
[G(\omega)]\{t\}=[F(\omega)] u .
$$

This system is finally partitioned according to the boundary conditions and is then solved for the unknown scattered displacements/tractions in terms of prescribed boundary values, all for a spectrum comprising $M$ discrete frequency values. Once the complete frequency spectrum is obtained, inverse FFT routines yield the solution in the time domain. 


\subsection{DeRiVATION OF IMPEDANCE Functions By THE BEM}

Impedance functions are formally defined as the force-displacement relationship at the soil-foundation interface DOF and can be represented as a frequency-dependent complex-valued stiffness matrix $[S(\omega)]$, where

$$
[S(\omega)] \cdot\{u(\omega)\}=\{P(\omega)\}
$$

with $\{u(\omega)\}$ and $\{P(\omega)\}$ being the displacement and load vectors, respectively. In general, impedance functions at a given DOF can be expressed in complex form as

$$
S_{i j}(\omega)=K_{i j}(\omega)+i \omega C_{i j}(\omega),
$$

where $\omega$ is the excitation frequency, $K_{i j}(\omega)$ are the dynamic stiffness coefficients (corresponding to springs) and $C_{i j}(\omega)$ are the dynamic damping coefficients (corresponding to dashpots). It is noted here that damping, as computed by the BEM, represents both energy dissipation due to outgoing waves from the foundation (radiation damping), as well as material damping in the soil medium itself.

Following conventional definitions, impedance functions are produced for rigid massless foundations, i.e. for a BVP involving the soil continuum with a rigid finitesized boundary placed along the soil-foundation interface (see Fig. 1). As such, in order to recover BEM impedance functions that can be compared to those available in the literature for strip foundations, unit displacements $u_{i}$ are imposed along this finite soil-foundation interface. The remaining horizontal surface is traction-free and the solution reproduces the rigid foundation's response to three possible types of motion: vertical, horizontal and in-plane rotation. More specifically, the integration of

Rigid embedded foundation

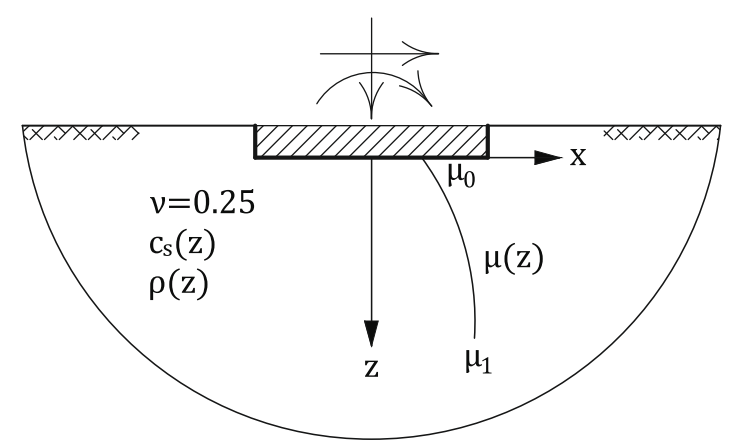

Fig. 1: Rigid foundation embedded in inhomogeneous soil under imposed unit displacement states. 
the resulting traction $t_{i}$ profile along the length of the boundary yields complex stiffness functions defined at the centroid of the rigid foundation, a procedure that must repeated for every frequency value $\omega$. We note in passing that in order to reproduce a flexible foundation, then the BVP changes and the imposed loading would have to be unit tractions along the foundation length, with the remaining horizontal surface being traction-free. As far as the kinematic boundary conditions are concerned, the ends of the surface mesh must either be fixed, or infinite elements must be placed there, which have the added advantage of moving the fixity condition to (theoretically) infinity. Also, symmetry conditions can be imposed at the origin if warranted. For instance, for a vertical loading, the node at the origin of the coordinates has a zero horizontal displacement.

For the problem under consideration, as shown in Fig. 1, the following three cases are distinguished: (i) imposition of a vertical unit displacement at the soil-foundation interface, where the resulting traction distribution $t_{Z}$ corresponds to the vertical com-
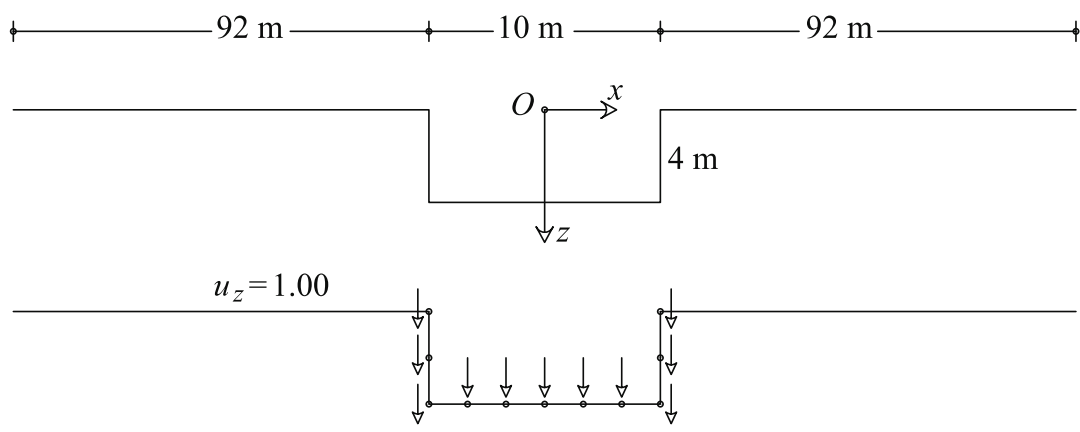

(b)

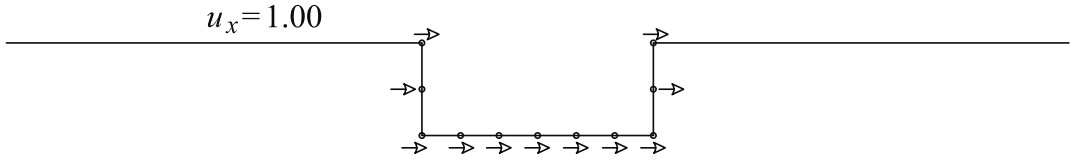

(c)

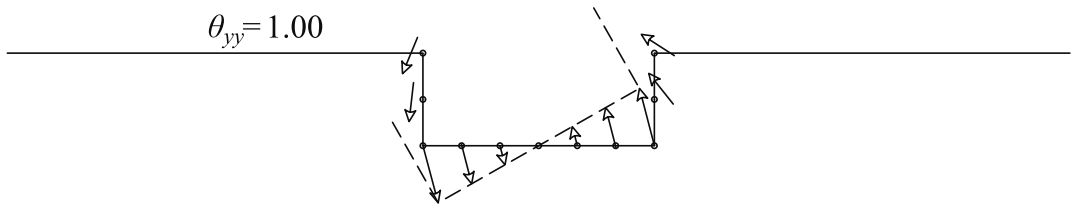

Fig. 2: (a) Three kinematic states in the BVP for the embedded foundation leading to the recovery of (b) vertical; (c) horizontal and (d) coupled rotational impedance functions. 
plex impedance $S_{Z Z}(\omega)$; (ii) imposition of a horizontal unit displacement, where the traction distribution $t_{X}$ corresponds to the horizontal complex impedance $S_{X X}(\omega)$; (iii) imposition of a unit rotation about the center of the foundation, where the traction distribution $t_{Z}$ corresponds to the complex rocking impedance $S_{\Theta \Theta}(\omega)$ and the traction distribution $t_{X}$ corresponds to the coupled translational-rotational impedance $S_{\Theta X}(\omega)=S_{X \Theta}(\omega)$. This rotational displacement is reproduced by imposing linearly varying displacements in opposite directions along the two halves of the foundation length. If an embedded foundation is considered, displacements along the vertical sides must be added, since imposition of a unit rotation about the center must preserve the original shape of the foundation. These three kinematic states for the embedded foundation case are depicted in Fig. 2 for clarity. Finally, the complex impedance matrix at the centroid of the strip foundation has the following form:

$$
S(\omega)=\left[\begin{array}{ccc}
S_{Z Z} & 0 & 0 \\
0 & S_{X X} & S_{X \Theta} \\
0 & S_{X \Theta} & S_{\Theta \Theta}
\end{array}\right] .
$$

\section{The LefKas Island Municipality Building CASe Study}

In order to illustrate the recovery of soil impedance functions in engineering practice, a case study involving a low-rise, municipal building located in the island of Lefkas, Greece (see Fig. 3) is presented. The importance of this case study stems from the fact that the building was instrumented with a special accelerometer array in 2012 by the Institute of Engineering Seismology and Earthquake Engineering (ITSAK), the research division of the Earthquake Planning and Protection Organization of Greece (EPPO), in order to record its response to seismic excitations. Additionally, a highprecision accelerograph was installed by ITSAK under virtually free-field conditions, at a distance of approximately $40 \mathrm{~m}$ from the building in 2013. This arrangement turns out to be a good benchmark case for examining SSI phenomena, since the external recording is practically the surface manifestation of the ground input motion to the structure [25]. Previous research efforts aimed at examining the discrepancies between the motions recorded by the free-field accelerograph and those recorded at the base of the examined structure [26], as well as of an adjacent building [27, 28], clearly demonstrated the presence of SSI phenomena at this particular site.

It is also worth noting that the Lefkas municipality building has a structure to soil stiffness ratio of approximately 0.27 , which suggests significant SSI effects can be expected, a fact that has been confirmed from the analysis of recordings during recent earthquake events. This structure to soil stiffness ratio is defined as $h \cdot f / V_{s}$ (also denoted as $1 / \sigma$ ), where $h$ is estimated as $2 / 3$ of the building's height from foundation to roof [29], $f$ is the first eigenfrequency of the structure under fixed-base conditions and $V_{s}$ the time-averaged shear wave velocity of the soil at an appropriate 


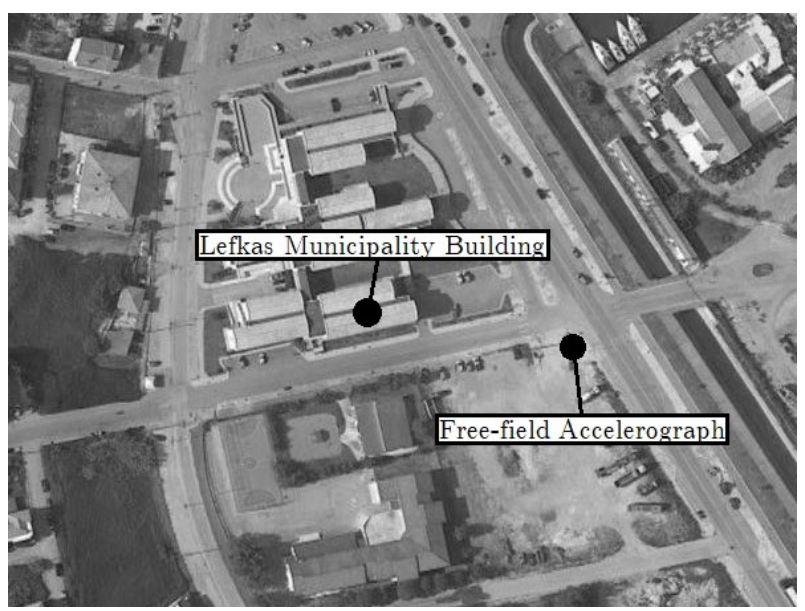

(a)

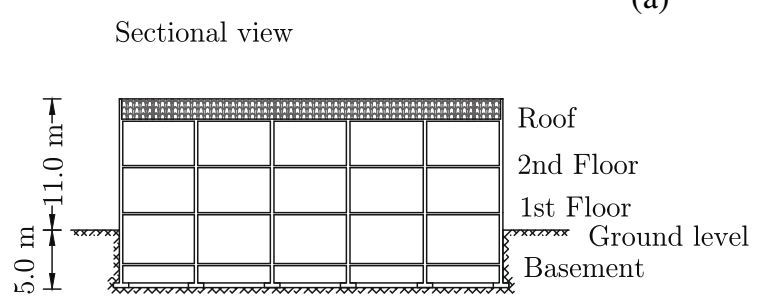

(b)
Plan view

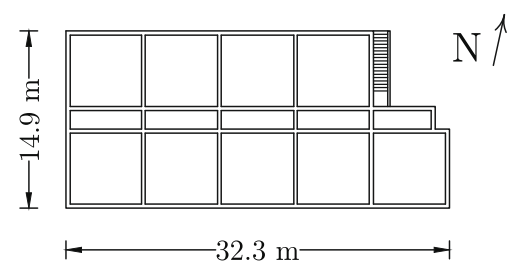

Fig. 3: (a) The Lefkas island, Greece, municipality building and the location of the free-field accelerograph and (b) section and plan views of the two-story R/C frame building.

depth. This parameter is used to gauge the significance of inertial SSI effects on simple structures founded on soft ground [30], despite shortcomings associated with its use [31]. In sum, ratio values higher than 0.1 typically indicate important SSI effects. The characterization of the soil profile at the building site was based on data obtained from geotechnical tests conducted at a nearby location [32]. The crosssection of the soil profile, which consists of alluvial sediments overlying sedimentary rock, as well as the shear modulus variation with depth are all presented in Fig. 4. The average shear wave velocity of the soil at the first $30 \mathrm{~m}$ is $V_{s}=346.8 \mathrm{~m} / \mathrm{s}$, which results in a type $\mathrm{C}$ soil profile according to the Eurocode 8 site classification system.

For comparison purposes, the estimation of the impedance functions was based on both the proposed BEM computational methodology, as well as the methodology described in the NIST/GCR 11-917-14 report [28], which makes use of the formulas 


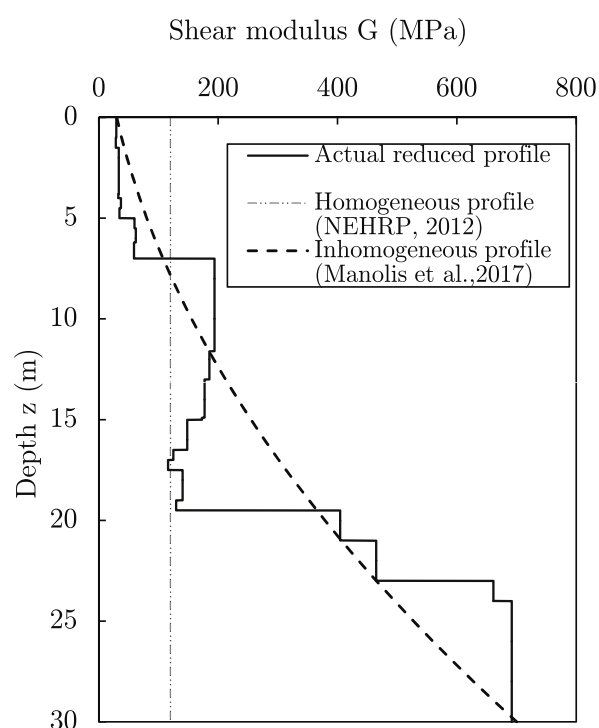

(a)
Soil profile

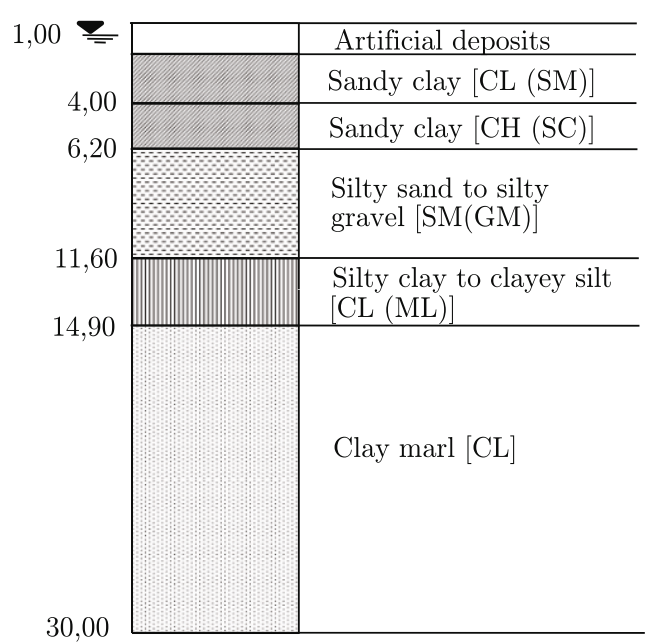

(b)

Fig. 4: Borehole data from the Lefkas municipality building site geotechnical prospecting: (a) soil shear modulus profile plus an approximate smooth variation with depth and (b) soil stratigraphy.

developed byPais and Kausel [8] and by Mylonakis et al. [21]. It is noted here that the impedance functions are not only a function of the building type and the soil characteristics, but also of the intensity of the earthquake excitation, due to the non-linear behavior of the soil. In the present study, the impedance functions were calculated for a M6.4 earthquake event that occurred on 17/11/2015, approximately $23.34 \mathrm{~km}$ southwest from the Lefkas Island. In order to account for the non-linear behavior exhibited by the soil, which gives variable shear-wave velocity profiles over time that are difficult to implement in transient analyses, a reduced shear-wave profile was used. The estimation of this reduced profile was based on the calculation of the average shear strain for each soil stratum during the earthquake, followed by an average reduction of the shear modulus through use of appropriate shear modulus-shear strain-depth $(G-\gamma-d)$ curves [33]. Because neither methodology can account explicitly for soil inhomogeneity, two distinct approximations for the soil profile in question were made: (i) An inhomogeneous shear modulus profile varying as a quadratic function of depth was considered in the BEM computational model and (ii) an averaged homogeneous shear modulus profile at an appropriate depth, which is a function of the building's foundation dimensions [29], was considered for the use of the literature 
formulas. The profiles that were used for each methodology are presented in Fig. 4a. Since the proposed BEM can only account at present for plane strain conditions, whereby one foundation dimension is very large compared to the others, a 2D model of the foundation was considered (i.e. strip foundation).

\subsection{BEM COMPUTATIONAL MODEL}

As previously mentioned, the computation of the soil impedance functions was done by the displacement-based BEM with a frequency-dependent fundamental solution obtained in closed form for continuous soil inhomogeneity [3]. The BVP consisted of imposed prescribed unit displacements at the soil-foundation interface, while the remaining horizontal surface was traction free. The soil shear modulus profile varied as a quadratic function of depth, as shown in Fig. 4a, and was calculated via a least-squares fitting approach to the actual profile. The same approach was used to calculate the mass density profile that also varied as a quadratic function of the depth, while the Poisson's ratio was considered constant at $\nu=0.25$. In order to include viscoelasticity in the soil material, the shear modulus was redefined as a complex variable in the form $\mu_{V}=\mu(1+i \eta \omega)$, where the value of the coefficient of viscosity is taken as $\eta=1 \%$.

The examined strip foundation was the closest possible representation of the actual foundation of the Lefkas municipality building, see Fig. $4 \mathrm{~b}$. The foundation was centrally placed on the free surface of the soil, which is modeled to a total lateral distance of $204 \mathrm{~m}$, see Fig. 2. For the BEM discretization of the entire horizontal surface, three-node, quadratic line boundary elements (BE) were used. For the embedded strip foundation, the BEM mesh comprised $101 \mathrm{BE}$, resulting in 203 nodes and 406 DOF. Furthermore, the two BE at the left and right end the surface mesh were infinite BE. These have modified shape functions that map their outer node to infinity, which serves as an indirect way for extending the BEM mesh. Next, the range of the external frequencies of vibration of the strip foundation for a double-sided Fourier transform goes up to $10 \mathrm{~Hz}$, split as $-5.0 \mathrm{~Hz}<f<5.0 \mathrm{~Hz}, f=2 \pi / \Omega$, and swept in 128 increments. This corresponds to a time scale of $T=6.4 \mathrm{~s}$, and to a time increment $d t=0.1 \mathrm{~s}$. In order to gauge the extent of the surface BEM mesh, as well as the length of the BE used, we note that the wavelength for the shear waves in the soil medium $\lambda_{S}=2 \pi V_{S} / \omega$ is $62.6 \mathrm{~m}$ when the upper limit $\omega=\Omega$ is reached. Thus, the BEM mesh is judged to be satisfactory and the area occupied by the foundation will not see reflections until well past the end of time interval $T$. Regarding the size of a typical BE length, we consider a representative shear wave speed of $V_{S}=350 \mathrm{~m} / \mathrm{s}$ and a maximum frequency value of $\omega=60 \mathrm{rad} / \mathrm{s}$. This gives a reference wavenumber of $\kappa_{S}=\omega / V_{S}=0.17 \mathrm{~m}^{-1}$, corresponding to a reference wavelength of $\lambda_{S}=2 \pi / \kappa_{S}=37.7 \mathrm{~m}$. So long as the BE length remains much less 
than that, and here it is $l_{B E}=2 \mathrm{~m}$, the basic accuracy of the spatial discretization is assured. Finally, a rudimentary convergence study was also carried out, which indicated that past $50 \mathrm{BE}$ for the soil-foundation surface discretization, results for the traction distribution at the surface converged.

\section{Results AND Discussion}

As previously mentioned, the dynamic impedance functions for the present case study were computed by the proposed BEM model, as well as by the formulas given in Pais and Kausel [8] and in Mylonakis et al. [21]. The former formulas [8] constitute approximate formulas for describing the variation of the soil's impedance matrix with frequency, obtained by fitting mathematical expressions to numerical solutions from FEM and BEM analyses of a homogeneous half-space. Likewise, the latter formulas [21] are based on solutions originally obtained by Gazetas [9], but improved with minor adjustments in some of the coefficients, and are for arbitrarily shaped foundations resting on or embedded in an elastic, homogeneous half-space. The first set of results, which is plotted in Fig. 5, concerns the stiffness component of the dynamic impedance functions of an embedded foundation, as computed for the Lefkas municipal building. More specifically, these are functions of the frequency $f$, following conversion from the dimensionless frequency $a_{0}=\omega B / V_{s}$, where $B$ is the foundation half-width, and are defined at the center of the embedded foundation. At first, good agreement is observed between the two solutions recovered from the literature, which $[8,21]$ corresponding to the equivalent homogeneous soil profile, except for the coupled rocking-horizontal component, where the divergence between them reaches a maximum value of about $15 \%$. Regarding the BEM-derived coefficients for the inhomogeneous soil profile, it is expected that they will be different from the engineering approximations. More specifically, the vertical stiffness component $K_{Z Z}$ is approximately $1 / 3$ times greater than the values obtained by the solutions from the literature. On the other hand, the numerical values for the stiffness components corresponding to the remaining DOF are smaller, with maximum differences observed for the rocking and the coupled rocking-horizontal translation components, namely $K_{\Theta \Theta}$ and $K_{\Theta X}$. The values for these differences range at around $30 \%$ and $60 \%$, respectively. It is important to note that the BEM-derived coefficients came from the integration of the interface traction distribution so as to produce values lumped at the centroid of the foundation.

These differences in the soil stiffness values are partially attributed to the complex pressure distributions across the soil-foundation interface, which are highly dependent on frequency and this may cause numerical difficulties. Moreover, discrepancies can be attributed to the different pressure distributions in the soil for each DOF and the resulting different "effective" depths, for which the average shear modulus 


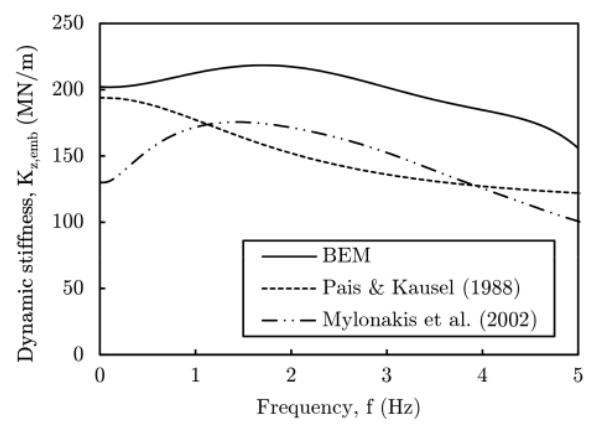

(a)

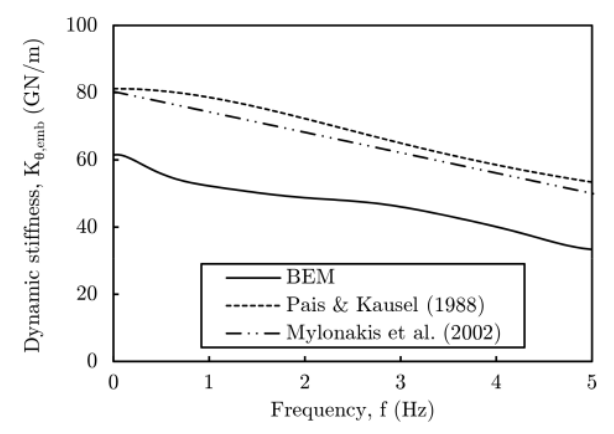

(c)

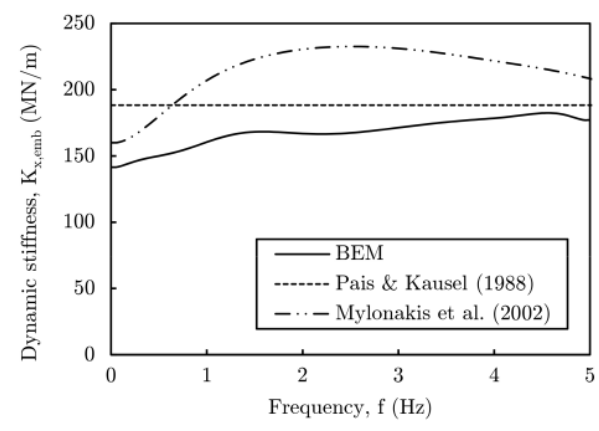

(b)

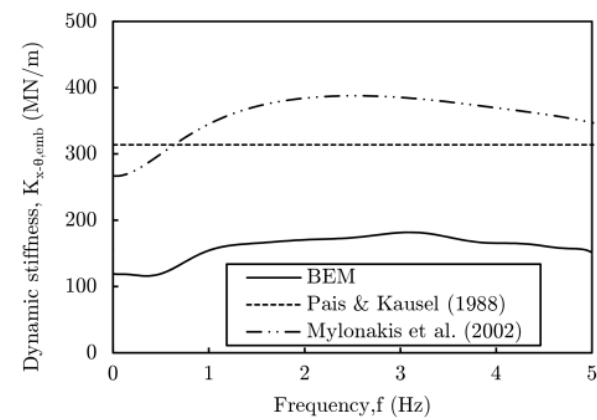

(d)

Fig. 5: Frequency dependent soil stiffness coefficients for the Lefkas municipality building foundation: (a) vertical, (b) horizontal, (c) rotational and (d) coupled horizontal-rotational components.

of the inhomogenous soil profile coincides with the constant shear modulus of the homogenous profile. It is thus impossible to choose a single appropriate "effective" shear modulus for the soil profile, which is inherently inhomogenous. For the vertical stiffness component, the pressure distribution ("pressure bulb" concept) reaches greater depths, which results in greater stiffness values. On the other hand, the stiffness coefficients for the other DOF are affected primarily by the upper soil layers, thus resulting in lower stiffness values. However, it can be stated that the average shear modulus value used in this case study as per the NIST recommendations [27] is satisfactory from an engineering point of view.

The damping components of the computed impedance functions for the present case study are presented in Fig. 6. As before, a relatively good agreement between the two literature-based solutions is observed, with differences occurring for low fre- 


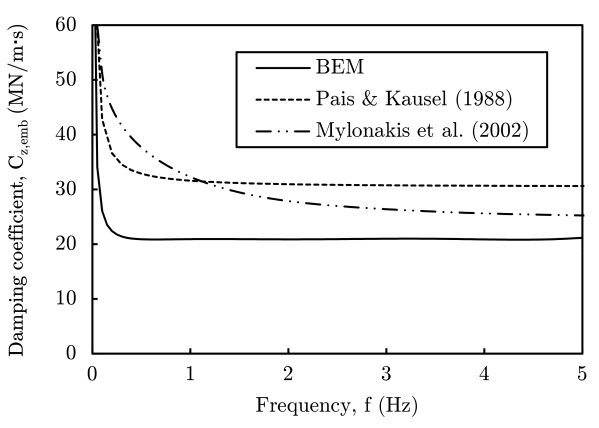

(a)

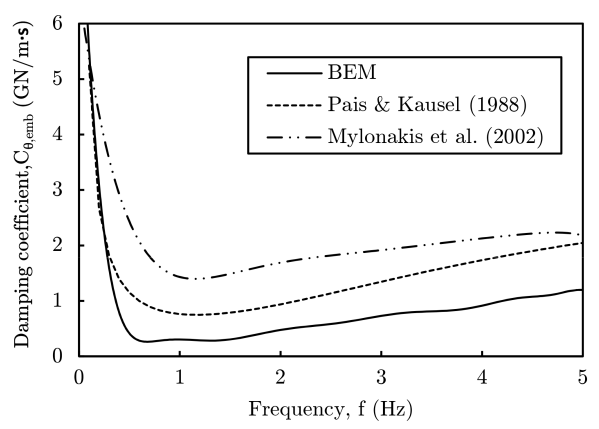

(c)

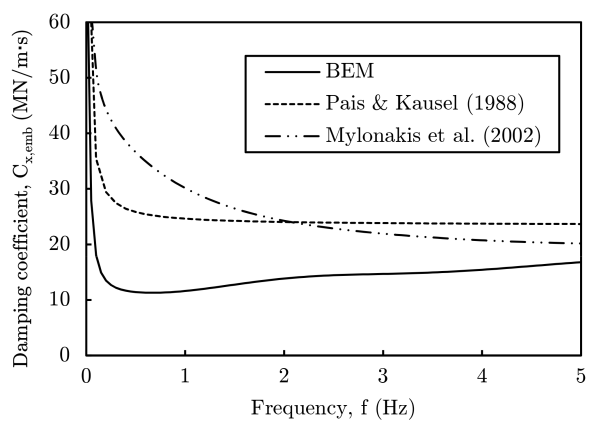

(b)

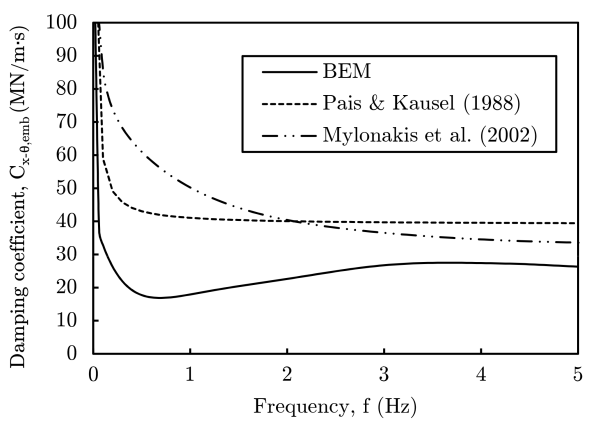

(d)

Fig. 6: Frequency dependent damping coefficients for the Lefkas municipality building foundation: (a) vertical, (b) horizontal, (c) rotational and (d) coupled horizontalrotational components.

quencies, less than 1.0 Hz. Greater discrepancies are again observed between the BEM-derived and the literature-based solutions, especially in the lower frequencies, where the BEM-derived damping coefficient values are significantly smaller. This can be partly attributed to the reduced radiation damping exhibited by inhomogeneous soil profiles, where elastic waves are continuously reflected and refracted, resulting in complex wave fields in the near field around the foundation and thus a less effective dissipation of energy with comparison to a homogeneous medium. Another reason is the definition of damping, whereby different forms of material damping may be introduced in the solutions. In our case, structural damping based on the correspondence principle of viscoelasticity is used in the BEM. However, given all these uncertainties, the use of an equivalent homogenous soil profile may be deemed sufficiently accurate from an engineering point of view, depending of course on the 
requirements of each specific project.

In closing, it is important to underline that the derivation and use of impedance functions to model the soil continuum in SSI studies is associated with several shortcomings. First of all, potential secondary modes of the foundation's vibrations, which result from geometric asymmetries, are ignored. For example, a vertical unit displacement of the foundation may result in a non-uniform traction distribution along the soil-foundation interface, which will cause an additional rotational movement. These secondary modes become even more pronounced for flexible foundations, where the relative stiffness of the foundation to the soil may significantly alter the results. The same holds true if the mass of the foundation is taken into account. In addition, geometric non-linearities, such as foundation uplift or sliding, cannot be readily modeled by this approach. Finally, several aspects concerning the material behavior of the soil (e.g., non-linearities, discontinuities) are also difficult to implement. As such, use of impedance functions in their classical sense may be more appropriate in the early design stages, where high modeling accuracy is not of primary importance. In other cases, detailed direct approaches for modeling the SSI system may be more suitable. An efficient approach is the use of hybrid modeling techniques, where the BEM is used to represent the semi-infinite soil medium and the results are re-casted in as a macro finite element to be used directly in commercial FEM packages [34]. Via such an approach, the complexities of the SSI system can be taken into account in a rational way, while retaining at the same time the basic advantage of efficiency and accuracy in the modeling process.

\section{CONCLUding RemarKS}

A BEM computational methodology for the determination of the dynamic impedance functions of arbitrarily shaped, embedded strip foundations in inhomogeneous soil media was presented. The aforementioned methodology was used in conjunction with a case study of a well-documented municipal building in the Lefkas island so as to illustrate the difficulties associated with the derivation of impedance functions and to identify the challenges associated with this procedure. The following points can be summarized:

(i) While good agreement was observed between the two groups of literaturebased impedance functions for the examined case study, the agreement between them and the more rigorously computed impedance functions by the BEM exhibited discrepancies. This is to be expected, given the differences in the underlying theoretical background and the various assumptions that were necessary in the derivations.

(ii) Regarding the BEM stiffness components, the vertical spring values were found to be greater, while the horizontal, rotational, and coupled horizontal-rotational spring values were found to be smaller, as compared to the literature-based impedance 
functions. The basic reason for this can be traced back to the equivalence between homogeneous and inhomogeneous soil representations, where in the former case different soil depths (the 'pressure bulb' concept) need to be assumed for each loading state.

(iii) Regarding the damping components, the values computed by the BEM were found to be significantly lower than their engineering-type counterparts, which indicate low radiation damping for the inhomogeneous soil. This is so because of the dispersion phenomena from the continuously changing material parameters of the soil continuum that inhibits elastic wave radiation.

(iv) The average shear modulus value used for the determination of the impedance functions as per the NIST recommendations [28], was deemed satisfactory from an engineering point of view, subject of course to the particular requirements of each SSI case examined.

(v) Sub-structuring, whereby the soil and foundation components of the complete structural system are separately treated, has the basic advantage that the semi-infinite soil continuum is represented by a set of springs and dashpots. Their determination and use, however, entails several challenges associated not only with the geometric irregularities and the flexibility of the foundation, but also with the complexities of the soil continuum, such as its configuration, its non-linear behaviour, ground discontinuities, surface topography, etc., that cannot be explicitly accounted for despite recent research efforts.

(vi) The alternative of introducing 'macro-elements', which essentially encompass semi-analytical or numerical solutions using efficient methods for the soil continuum such as the BEM, presents a promising alternative for more accurate and efficient engineering simulations, as they can be included in the FEM program library and then added to the finite element model of the superstructure. This obviates the need to define in an ad hoc way springs and dashpots lumped at various control points along the soil-foundation interface.

(vii) In closing, it seems necessary to conduct either field measurements or laboratory experiments of foundation slabs resting on soil deposits to gauge as many of the assumptions that were listed in this work as possible in terms of their relative importance.

\section{ACKNOWLEDGEMENTS}

The authors wish to acknowledge support provided through the German Federal Ministry of Education and Research (BMBF) Grant No. IB-RA2014-178, Infrastructure Vulnerability under Environmentally-Induced Loads: Hybrid 3D Modelling of Underground Structures in Complex Geological Formations, Professor Frank Wuttke, University of Kiel, Coordinator. 


\section{REFERENCES}

[1] J.P. Wolf (1985) "Dynamic Soil-Structure Interaction". Prentice Hall, New Jersey.

[2] J.D. Achenbach (1973) "Wave Propagation In Elastic Solids". North-Holland, Amsterdam.

[3] G.D. Manolis, P.S. Dineva, T.V. Rangelov, F. Wuttke (2017) "Seismic Wave Propagation in Non-Homogeneous Elastic Media by Boundary Elements". Springer, Cham; DOI: 10.1007/978-3-319-45206-7.

[4] E. KAUSEL (2010) Early history of soil-structure interaction. Soil Dynamics and Earthquake Engineering 30 822-832; DOI: 10.1016/j.soildyn.2009.11.001.

[5] J.J. Johnson (1981) Soil-Structure-Interaction: The Status of Current Analysis Methods and Research. Research Report NUREG CR-1780. Washington, DC.

[6] B. Vivek, P. Raychowdhury (2017) Influence of SSI on period and damping of buildings supported by shallow foundations on cohesionless soil. International Journal of Geomechanics ASCE 17 04017030; DOI: 10.1061/(ASCE)GM.1943-5622.0000890.

[7] A.S. Veletsos, Y.T. WeI (1971) Lateral and Rocking Vibration of Footings. Journal of the Soil Mechanics and Foundations Division ASCE 97 1227-1248.

[8] A. PAis, E. Kausel (1988) Approximate formulas for dynamic stiffnesses of rigid foundations. Soil Dynamics and Earthquake Engineering 7 213-227; DOI: 10.1016/S0267-7261(88)80005-8.

[9] G. Gazetas (1991) Formulas and Charts for Impedances of Surface and Embedded Foundations. Journal of Geotechnical and Geoenvironmental Engineering ASCE 117 1363-1381; DOI: 10.1061/(ASCE)0733-9410(1991)117:9(1363).

[10] J.-G. SiefFert, F. Cevaer (1992) "Handbook of Impedance Functions: Surface Foundations". Ouest Editons, Nantes.

[11] J.P. Wolf (1998) Simple physical models for foundation dynamics. Developments in Geotechnical Engineering 83 1-70; DOI: 10.1016/S0165-1250(98)80004-7.

[12] J.P. Wolf, A.J. DeEKs (2004) "Foundation Vibration Aanalysis: A Strength-ofMaterials Approach". Elsevier, Amsterdam.

[13] G.D. MANOLIS, D.E. BeSKos (1981) Dynamic stress concentration studies by boundary integrals and Laplace transform. International Journal for Numerical Methods in Engineering 17 573-99; DOI: 10.1002/nme.1620170407.

[14] S. Kobayashi, N. Nishimura (1983) Analysis of Dynamic Soil-structure Interactions by Boundary Integral Equation Method. In: P. Lascaux (ed) "Numerical Methods in Engineering". Pluralis, Paris, pp. 353-362.

[15] R. Abascal, J. Domínguez (1986) Vibrations of footings on zoned viscoelastic soils. Journal of Engineering Mechanics ASCE 112 433-447; DOI: 10.1061/(ASCE)0733-9399(1986)112:5(433).

[16] F.T. Kokkinos, C.C. Spyrakos (1991) Dynamic analysis of flexible stripfoundations in the frequency domain. Computers \& Structures 39 473-482; DOI: 10.1016/0045-7949(91)90055-Q. 
[17] D.L. Karabalis, D.C. Rizos (1993) Dynamic Analysis of 3D Foundations. 177-208 in G.D. Manolis, D.E. Beskos (eds) "Boundary Element Techniques in Geomechanics". Elsevier, London.

[18] J. QIAN, D.E. BESKos (1995) Dynamic interaction between 3-D rigid surface foundations and comparison with the ATC-3 provisions. Earthquake Engineering and Structural Dynamics 24 419-437; DOI: 10.1002/eqe.4290240309.

[19] Z. BA, J. LIANG, V.W. LEE, L. HU (2018) IBEM for Impedance Functions of an Embedded Strip Foundation in a Multi-Layered Transversely Isotropic Half-Space. Journal of Earthquake Engineering 22 1415-1446; DOI: 10.1080/13632469.2017.1286621.

[20] D. Pitilakis, A. Moderessi-Farahmand-Razavi, D. Clouteau (2013) Equivalent-Linear Dynamic Impedance Functions of Surface Foundations. Journal of Geotechnical and Geoenvironmental Engineering 139 1130-1139. DOI: 10.1061/(ASCE)GT.1943-5606.0000829.

[21] G. Mylonakis, G. Gazetas, S. Nikolaou, A. Chauncey (2002) Development of Analysis and Design Procedures for Spread Footings. Technical Report MCEER-020003. University at Buffalo, New York.

[22] J.P. BARDET (1992) A viscoelastic model for the dynamic behavior of saturated poroelastic soils. Journal of Applied Mechanics ASME 59 128-135; DOI: 10.1115/1.2899417.

[23] T.V. Rangelov, G.D. Manolis (2010) Time-harmonic Green's function for the halfplanewith quadratic-type inhomogeneity. 147-160 in E.J. Sapountzakis (ed) "Recent Develpoments in Boundary Element Methods". WIT Press, Southampton.

[24] G.D. Manolis, T.V. Rangelov, P.S. Dinev (2007) A Free-field Wave Solutions in a Halfplane Exhibiting a Special-type of Continuous Inhomogeneity, Wave Motion 44 304-321.

[25] R. Villaverde (2009) "Fundamental Concepts of Earthquake Engineering". CRC Press, Boca Raton; DOI: 10.1201/9781439883112.

[26] C.Z. Karakostas, E.N. Rovithis, K.E. Morfidis, G.A. Chatzistefanou, V.A. LEKIDIS, N.P. THEODOULIDIS (2018) Investigation of the dynamic response and SSI effects of the instrumented municipality building in Lefkas, Greece. 16th European Conference in Earthquake Engineering (16ECEE), 18-21 June 2018, Thessaloniki, Greece.

[27] D. Sotiriadis, N. Klimis, B. Margaris, A. Sextos (2019) Influence of structure-foundation-soil interaction on ground motions recorded within buildings. Bulletin of Earthquake Engineering 17 5867-5895; DOI: 10.1007/s10518-019-00700-6.

[28] C.Z. Karakostas, G. Kontogiannis, K.E. Morfidis, E. Rovithis, G.D. MANOLIS, N.P. THEODOULIDIS (2017) Effect of soil-structure interaction on the seismic response of an instrumented building during the Cephalonia, Greece earthquake of 26-1-2014. In: Proceedings of the 6th International Conferenceon Computational Methods in Structural Dynamics and Earthquake Engineering (COMPDYN 2017) 1, National Technical University, Athens. 1375-1387. DOI: 10.7712/120117.5499.17812.

[29] NEHRP Consultants Joint Venture (2012) Soil-Structure Interaction for Building Structures. NIST GCR 12-917-21 Report, Gaithersburg, MD. 
388 BEM-Derived Impedance Functions for 2D Inhomogeneous Soil Media: ..

[30] A.S. Veletsos, J.W. Meek (1974) Dynamic behaviour of building-foundation systems. Earthq Eng Struct Dyn 3 121-138; DOI: 10.1002/eqe.4290030203.

[31] X. Karatzia, G. Mylonakis (2019) On the applicability of Veletsos' wave parameter $(1 / \sigma)$. In: Proceedings of the 7th International Conferenceon Computational Methods in Structural Dynamics and Earthquake Engineering (COMPDYN 2019), Crete, Greece, pp. 1924-1932. DOI: 10.7712/120119.7046.19191.

[32] G. Gazetas, G. Koukis, A. Tselentis (2004) The 14.8.2003 earthquake of Lefkas - Collection/analysis of seismological, geological, geotechnical and structural data. Failure analysis of Lefkada, Ligia, Nidri and Vasiliki ports (in Greek), ITSAK-OASP Technical Report, Thessaloniki. ITSAK-OASP Publication.

[33] G.A. Chatzistefanou, C.Z. Karakostas, G.D. Manolis (2019) Investigation of the influence of dynamic soil-structure interaction on the seismic response of the instrumented municipality building of Lefkas, Greece (in Greek). In: Proceedings of the 4th Panhellenic Conference in Earthquake Engineering and Engineering Seismology (4 ETAM ), 5-7 Sept. 2019, Athens, Greece.

[34] M.B. Basnet, H.D.B. Aji, F. Wuttke, P. Dineva (2018) Wave propagation through poroelastic soil with underground structures via hybrid BEM-FEM. Zeitschrift Angew Math Mech (ZAMM) 98 1390-1411; DOI: 10.1002/zamm.201800023. 